\title{
Simultaneous bilateral atypical femoral fracture: case presentation
}

\begin{abstract}
Atypical femoral fractures are associated with the prolonged use of bisphosphonates, because they decrease bone remodeling and lead to the accumulation of micro fractures.

Simultaneous bilateral cases are very rare, more so with complete fractures and in the diaphyseal region. We report the case of a 55-year-old female with bilateral femur fracture in the diaphyseal zone. The osteosynthesis of both femurs was performed with endomedullary nails in the same act, both fractures presented delayed consolidation and a new intervention was necessary, the right femur was consolidated at 11 months, but the left femur progressed to pseudoarthrosis performs a third surgery, with the placement of a plaque, and consolidation is achieved 3 months later. This type of fracture has a slower healing, due to different factors, and a higher failure rate. New therapies are being studied to help achieve consolidation. Correct reduction of fracture is essential in these cases and many times, despite the correct treatment, new procedures are required to achieve consolidation.
\end{abstract}

Volume 9 Issue 5 - 2017

\author{
Francisco Schumacher, Federico Sartori, Luis \\ Vázquez \\ Servicio de Ortopedia y Traumatolog
}

Correspondence: Francisco Schumacher,Servicio de Ortopedia y Traumatologia, Sanatorio Allende, Cordoba, Argentina, Email franciscoeschmacher@gmail.com

Received: October 10, 2017 | Published: December 20, 2017

\section{Introduction}

Atypical femoral fractures (FFA) are defined as femoral stress fractures that comprise the diaphysis or the subtrochanteric region and which have certain characteristics, ${ }^{1}$ the term FFA was introduced to differentiate these fractures from osteoporosis.

This type of fracture is related to the use of bisphosphonates (BFN), the first report was published in 2005 by Odvina. ${ }^{2}$ In 2013 the American Society for Bone and Mineral Research (ASBMR) described the criteria for FFA diagnosis. These criteria were classified as major and minor (Table 1), ${ }^{1}$ at least 4 major criteria are needed for FFA diagnosis; juveniles are not needed but are usually present.

Table I Criteria for the diagnosis of atypical femoral fractures

\section{Major Criteria}

I. Located on any part of the femur, from the distal portion of the lesser trochanter to the proximal portion of the supracondylar prominence.

I. Associated with minimal trauma, such as falling while standing or at a lower height, or without previous trauma.

I. Transversal or short oblique configuration.

I. Without comminution.

I. Complete fractures extend through both cortices and may be associated with a medial spicule; incomplete fractures affect only the lateral cortex.

\section{Minor Criteria}

I. Localized periosteal reaction of the lateral cortex.

I. Generalized increase in the thickness of the cortex of the diaphysis.

I. Prodromal symptoms with dull or constant pain in thigh or inguinal.

I. Fractures and bilateral symptoms.

I. Delay of consolidation.

I. Associated diseases (eg, vitamin D deficiency, RA, hypophosphatasia).

I. Use of drugs (eg, bisphosphonates, glucocorticoids, PPIs).

Bisphosphonates are first-line anti-resorptive drugs used for the treatment of osteoporosis; several studies have proven effective in preventing vertebral and hip fractures. ${ }^{3-6}$ At present there is an increase in evidence that FFAs are related to the prolonged use of bisphosphonates. ${ }^{3,5-8}$ The incidence of these fractures is 110 per 100,000 people. ${ }^{8}$ The risk of fracture is higher with long-term treatment, over 5 years and decreases $70 \%$ per year since the last use. ${ }^{7,9}$

Although the pathogenesis is not well known these fractures are considered as stress fractures, BPN would suppress bone remodeling which would cause a deterioration of the bone micro architecture that would deteriorate the repair process and lead to an accumulation of micro fractures. ${ }^{69}$ Although rare, these lesions account for almost $9 \%$ of all "low-energy" femoral fractures. Therefore, in the presence of a "low energy" femoral fracture, we must have a high level of FFA suspicion. ${ }^{8}$

\section{Case}

We present the case of a female patient of 55 years who suffers a fall from the second step of a ladder which produced bilateral diaphyseal femur fracture.

Previous to the accident the patient reported pain in both thighs of 2 months of evolution, more in the right femur, according to the patient's report the pain had been progressive and increasing with the passage of days. She was being treated at another institution where she underwent radiography, magnetic resonance imaging (MRI) and total body scintigraphy (Figure 1). Both radiographs and MRI prior to trauma showed the presence of FFA and the scintigram reported increased bilateral uptake in femur diaphysis.

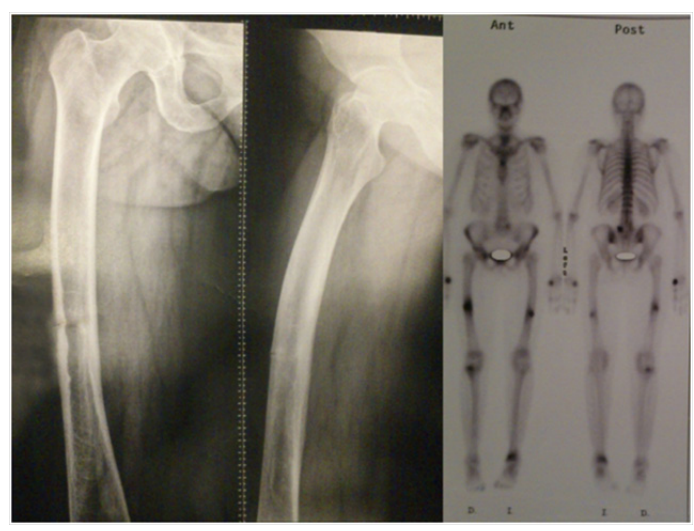

Figure I Preliminary studies of fractures.A) X-ray B) X-ray. 
The patient reports that she was not given any treatment for her condition, only the restriction of sports, but she continued with her usual medication and had been told to perform a biopsy. The patient was under treatment for osteopenia with vitamin $\mathrm{D}$ and alendronate for 6 years.

After the fall the patient is admitted and the closed fracture of both femurs in the diaphyseal region is verified, the fracture line was oblique short and with a medial spicule (Figure 2). Because of the characteristics of the radiographs and the anamnesis, we could verify that the patient presented 5 major criteria and 5 minor criteria for the diagnosis of FFA. The hemodynamic status was not compromised and presented no other problem.

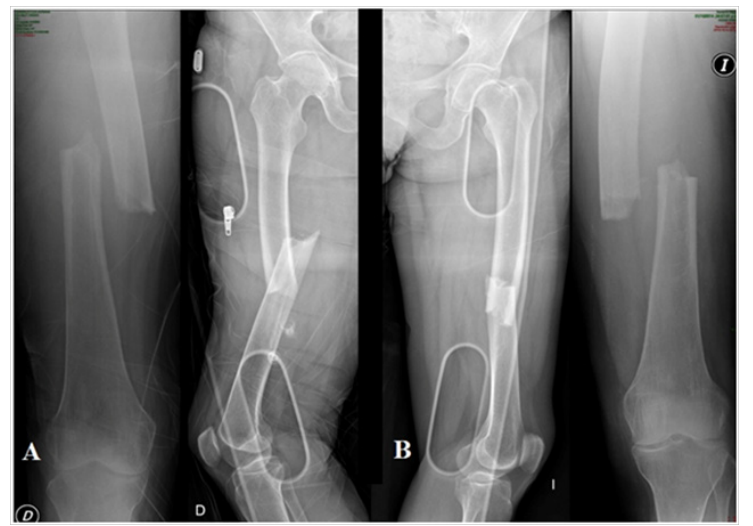

Figure 2 X-rays on admission. The characteristics of FFA are observed. A) Femur Der. B) Femur Left.

\section{Material and methods}

The patient was admitted to the common room and bilateral trans-tibial skeletal traction was performed. The reduction and osteosynthesis was performed on the same day of the accident, the patient was operated on a traction table and the osteosynthesis was performed on both femurs with milled intramedullary nails (Synthes universal nail). Osteosynthesis was performed first of the left femur and then the right one (Figure 3). Immediate partial load was indicated with the use of crutches. The use of alendronate was discontinued, it was indicated to continue with vitamin $\mathrm{D}$ and no other medication was added.

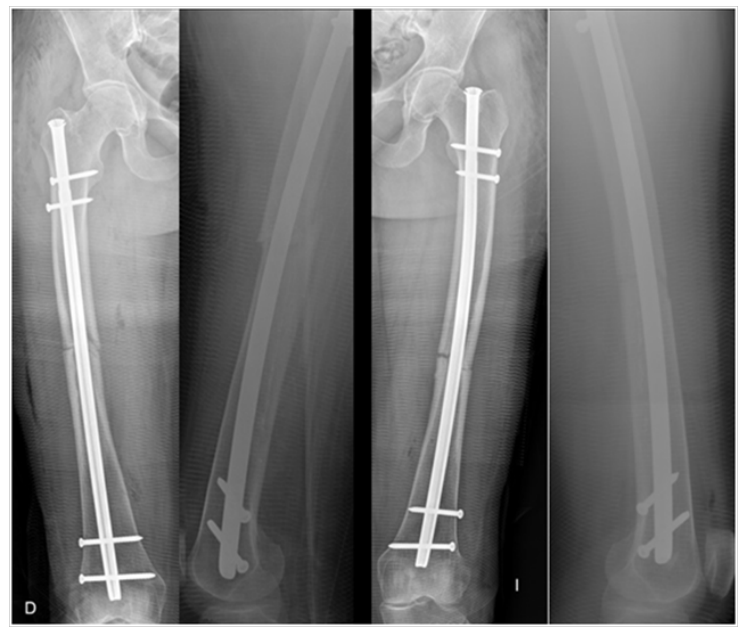

Figure 3 Immediate post-surgical radiographs.

The load without a walker was achieved after 3.5 months, but the patient used a cane because she reported pain in the left thigh.
At 6 months the patient wandered without help, with sporadic discomfort, the radiographs showed signs of consolidation and the patient gradually returned to their usual activities, but as activity increased, they began again with intermittent pain in the left thigh, new radiographs were taken and the patient was observed delay of the consolidation reason why the dynamization of both femurs is decided at eight months post surgical.

The consolidation of the right femur was completed at 11 months, no longer had pain or discomfort in the femur but continued with pain in the left thigh, new radiographs were made showing hypertrophic pseudoarthrosis so it was decided to perform a new surgery and place a plate blocked with minimally invasive technique on the nail, with this procedure immediate pain relief is achieved, full load was allowed after 2 weeks and consolidation was achieved 3 months later (Figure 4). Currently, the patient is 2.5 years old and does not present any pain or limitation in hip and knee mobility (Figure 5).

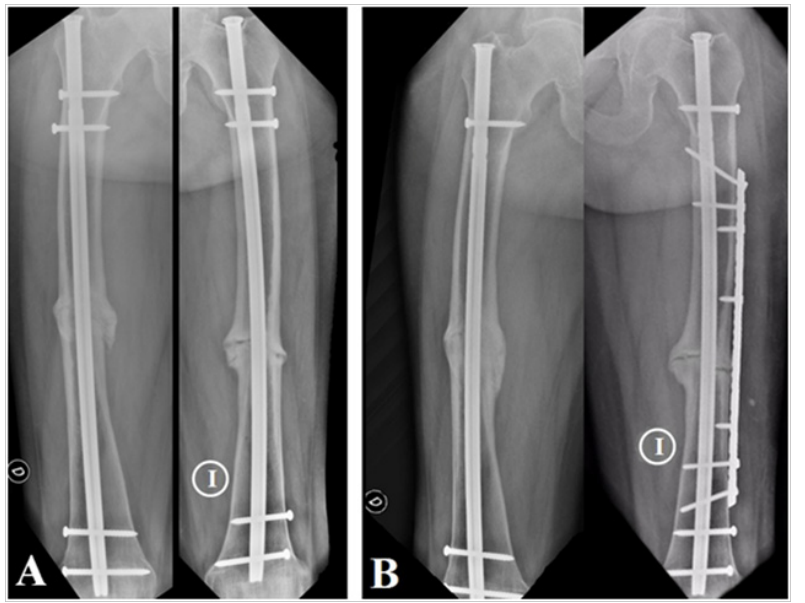

Figure 4 A) 6 months PO. B) II months PO: consolidation femur der. Plate MIO femur left.

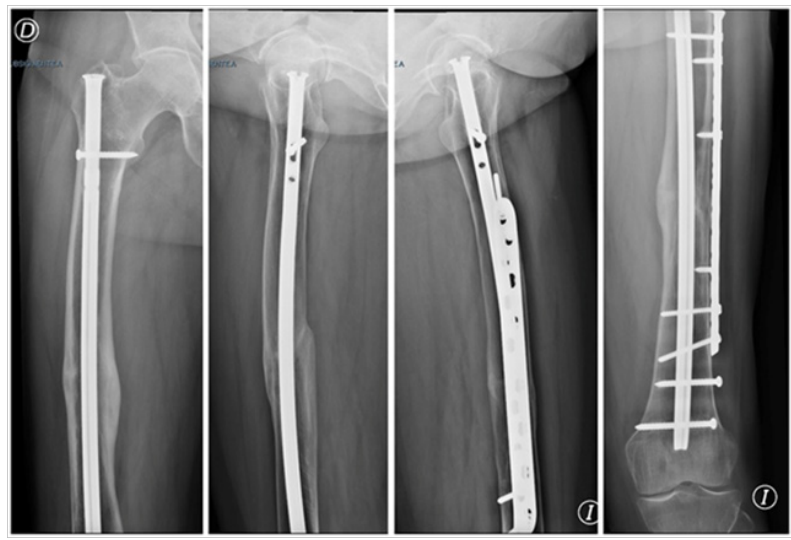

Figure $\mathbf{5}$ Consolidated fractures, 2 years post surgery.

\section{Discussion}

Bisphosphonates are undoubtedly an excellent treatment for osteoporosis and their effectiveness has been demonstrated. Its riskbenefit ratio is favorable; however, special attention must be paid to the duration of treatment. The intervals in therapy are necessary because the risk of FFA increases with its long-term use. ${ }^{6,710}$ This risk decreases rapidly after cessation of treatment.

The United States Food and Drug Administration (FDA) found no clear benefit from the long-term use (defined as more than 5 years) of bisphosphonates in the prevention of typical osteoporotic fractures. 
Black et al. ${ }^{5}$ however, suggest that there may be a benefit of continuing treatment for the subgroup of patients with persistent T-scores of less than -2.5 in the femoral neck after 3-5 years of treatment with bisphosphonates or those with vertebral fractures.

Patients presenting with hip or thigh pain for several months of evolution, especially if they are consuming LBW, should undergo $\mathrm{X}$-rays looking for an FFA. In the presented case the symptoms had begun several months before the fall. Our patient suffered fractures of both femurs in the diaphyseal region simultaneously, this type of fracture is rare even in patients with FFA, most of the fractures are not simultaneous, in the literature there are few cases of bilateral complete fractures, ${ }^{11-15}$ and As far as we know, our patient is the third case of bilateral simultaneous complete fracture in the diaphyseal region.

The treatment of choice for this type of fracture is the intramedullary nailing. In most studies it is recommended to use cephalomedicular nails, ${ }^{6,9,16-19}$ it is also worth mentioning that most of the FFAs are in the subtrochanteric region, in our patient the fractures were diaphyseal and it was decided to use universal nails for having them available at the institution and perform the surgery immediately.

The 2013 report of the ASBMR recommends the use of intramedullary nails, without specifying the type, and recommends avoiding the use of blocked plaques, also recommends suspending LBW and using calcium and vitamin D6, 8 supplements. Prasarn et al. ${ }^{18}$ observed a rate of implant failure of $30 \%$ with blocked plates compared to $0 \%$ with IM nails in AFF. ${ }^{18}$

Traditionally, the cephalomedullary nailing of subtrochanteric femur fractures is the standard with a cure rate of $96 \%$. In atypical femoral fractures, a recent retrospective study has shown excellent clinical results with cephalomedullary nails, reporting $98 \%$ healing. ${ }^{16}$ However Cho JW et al. ${ }^{16}$ in a study published this year reported a lower cure rate than previously published with $69 \%$ a year after surgery (the remaining 31\% required additional procedures) and that the healing time of subtrochanteric FFA treated with cephalomedullary nails was of an average of 10.7 months, significantly longer than that reported for standard subtrochanteric femur fractures, which heal in an average of 6 months. ${ }^{16}$ Recent studies that examined the surgical results of complete FFA treated with intramedullary nails, demonstrated a wide variability around complications and consolidation time ranging from 12 to $46 \% .^{17}$

Andrew J Lovy et al. ${ }^{17}$ reported that the use of concentrated bone marrow aspiration (BMAC) mixed with demineralized bone matrix placed percutaneously at the fracture site significantly decreased the time of union ( 3.5 vs 6.8 months, $p=0.004)$. They also reported that independently the poor reduction of the varus fracture led to a significant delay in consolidation time.

In our case, the left femur left us with a slight varus, we believe that because the femur of our patient was very curved and used as a point of entry the fossil happened that complication, when we performed the right femur, we decided to start with the trochanter to avoid varus reduction. This reduction in varus of the left femur we believed was tolerable at that time, but later evolved to pseudoarthrosis with which we had to perform a new surgery. The consolidation time of the right femur was 11 months similar to that published in the literature for this type of fracture. ${ }^{17}$

In the left femur we decided to use a plate on the nail already placed because we saw the formation of hypertrophic callus, the consolidation was achieved 3 months after this last surgery.

Although most of the literature does not support the use of plates in $\mathrm{FFA}^{6,8,18}$ its use is described in incomplete fractures with excellent results, another serious indication in very curved femurs where the placement of a nail would be very difficult or with great risk of iatrogenic fracture and in very narrow femurs. ${ }^{20}$

In the mentioned study, it was used together with osteosynthesis as adjuvant Teriparatide in a systemic way.

We do not indicate any additional medication with our patient. Currently there is an increase in the evidence in the use of bone anabolics, the most studied is Teriparatide (recombinant form of parathyroid hormone) that shows promising results to increase callus formation and accelerate consolidation. ${ }^{6,16,21-24}$ So far there is not enough evidence to recommend its use systematically, but it seems to have good results. In the same way, more clinical studies are needed. ${ }^{16}$ The use of this medicine is increasing for the conservative treatment of these fractures.

Both the ASBMR and MHRA (Medicines and Healthcare Products Regulatory Authority) provide guidance on the medical management of FFA that includes: the cessation of the antiresorptive; consider the use of Teriparatide in cases of lack of consolidation; ensure that the patient consumes calcium and vitamin D and performs radiography of the contralateral femur. ${ }^{8}$ The incidence of contralateral FFA is $19.3 \%$ in the following three years in patients who stopped taking BFN compared to $41.2 \%$ of those who continued taking them.

In case of incomplete fractures the treatment depends mainly on the associated symptoms. If the patient has pain in the thigh or groin, prophylactic surgery is advised. On the other hand, when the patient is asymptomatic, conservative treatment can be attempted for the first 2 or 3 months, with strict observation in order to quickly perform prophylactic surgery if signs of fracture progression or non-union appear.

In incomplete asymptomatic fractures associated with a simultaneous contralateral complete fracture, prophylactic surgery could be the gold standard to allow early loading, but the final decision depends on the patient's preferences. ${ }^{9,25}$

\section{Conclusion}

In summary, atypical femur fractures related to bisphosphonates are difficult to treat. This is due to a combination of factors that are the decrease of the curative potential by the use of bisphosphonates in the long term, the deformation forces of the proximal segment (subtrocanteric fractures) making the reduction difficult, the curvature of the femur (in general they are very femur curved) and high tension in the subtrochanteric and diaphyseal regions. The intramedullary nailing is the best option for the treatment of FFA (complete or incomplete) and also for prophylaxis. We have to bear in mind that there is a higher failure rate and that consolidation is slower in these patients. In our experience and for what is described in the bibliography, a precise reduction is essential in the treatment of these fractures, mainly the bad varus reduction should be avoided.

The use of adjuvants we believe may have a benefit but more studies are needed for its recommendation.

It is necessary to have a high index of suspicion of this pathology in patients with persistent pain in the thigh or groin to quickly and correctly treat this type of fractures before they are complete. Finally, we believe that multidisciplinary treatment is very important and would serve to improve the management of these patients.

\section{Acknowledgments}

None. 


\section{Conflicts of interest}

None.

\section{References}

1. Shane E, Burr D, Abrahamsen B, et al. Atypical subtrochanteric and diaphyseal femoral fractures: second report of a task force of the American society for bone and mineral research. $J$ Bone Miner Res. 2014;29(1):1-23.

2. Odvina CV, Zerwekh JE, Rao DS, et al. Severely suppressed bone turnover: a potential complication of alendronate therapy. $J$ Clin Endocrinol Metab. 2005;90(3):1294-1301.

3. Desai PA, Vyas PA, Lane JM. Atypical femoral fractures: a review of the literature. Curr Osteoporos Rep. 2013;11(3):179-187.

4. Van der Meulen MC, Boskey AL. Atypical subtrochanteric femoral shaft fractures: role for mechanics and bone quality. Arthritis Research \& Therapy. 2012;14(4):220.

5. Gedmintas L, Solomon DH, Kim SC. Bisphosphonates and Risk of Subtrochanteric, Femoral Shaft, and Atypical Femur Fracture: A Systematic Review and Meta-analysis. J Bone Miner Res. 2013;28(8):1729-1737.

6. Feron JM, Cambon-Binder A. Medication management after intramedullary nailing of atypical fractures. Injury. 2017;48Supp11:S15S17,

7. Schilcher J, Koeppen V, Aspenberg P, et al. Risk of atypical femoral fracture during and after bisphosphonate use: Full report of a nationwide study. Acta Orthop. 2015;86(1):100-107.

8. Eisenstein N, Kasavkar G, Bhavsar D, et al. Incidence and medical management of bisphosphonate-associated atypical femoral fractures in a major trauma center: a retrospective observational study. $B M C$ Musculoskelet Disord. 2017;18:29.

9. Toro G, Ojeda-Thies C, Calabrò G, et al. Management of atypical femoral fracture: a scoping review and comprehensive algorithm. $B M C$ Musculoskelet Disord. 2016;17:227.

10. Moghnie A, Scamacca V, De Fabrizio G, et al. Atypical femoral fractures bilaterally in a patient receiving bisphosphonate: a case report. Clin Cases in Miner Bone Metab. 2016;13(1):57-60.

11. Zafeiris CP, Stathopoulos IP, Kourkoumelis G, et al. Simultaneous bilateral atypical femoral fractures after alendronate therapy. $J$ Musculoskelet Neuronal Interact. 2012;12(4):262-264.

12. Higgins M, Morgan-John S, Badhe S. Simultaneous, bilateral, complete atypical femoral fractures after long-term alendronate use. J Orthop. 2016;13(4):401-403.
13. Capeci CM, Tejwani NC. Bilateral low-energy simultaneous or sequential femoral fractures in patients on long term alendronate therapy. J Bone Joint Surg Am. 2009;91(11):2556-2561.

14. Ovaska MT, Mäkinen TJ, Madanat R. Simultaneous bilateral subtrochanteric fractures following risedronate therapy. J Orthop Sci. 2011;16(4):467-470.

15. Puah KL, Tan MH. Bisphosphonate-associated atypical fracture of the femur: Spontaneous healing with drug holiday and re-appearance after resumed drug therapy with bilateral simultaneous displaced fractures - a case report. Acta Orthop. 201182(3):380-382.

16. Cho JW, Oh CW, Leung F, et al. Healing of Atypical Subtrochanteric Femur Fractures After Cephalomedullary Nailing: Which Factors Predict Union? J Orthop Trauma. 2017;31(3):138-145.

17. Lovy AJ, Kim JS, Di Capua J, et al. IM Nail Fixation of Atypical Femur Fractures with Bone Marrow Aspirate Concentrate Leads to Faster Union: A Case Control Study. J OrthopTrauma. 2017;31(7):358-362.

18. Prasarn ML, Ahn J, Helfet DL, et al. Bisphosphonate-associated femur fractures have high complication rates with operative fixation. Clin Orthop Relat Res. 2012;470(8):2295-2301.

19. Egol KA, Park JH, Rosenberg ZS, et al. Healing Delayed But Generally Reliable After Bisphosphonate-associated Complete Femur Fractures Treated with IM Nails. Clin Orthop Relat Res. 2014;472(9):2728-2734.

20. Tsuchie H, Miyakoshi N, Nishi T, et al. Combined Effect of a Locking Plate and Teriparatide for Incomplete Atypical Femoral Fracture: Two Case Reports of Curved Femurs. Case Rep Orthop. 2015;213614.

21. Ellegaard M, Jørgensen N, Schwarz P. Parathyroid hormone and bone healing. Calcif Tissue Int. 2010;87(1):1-13.

22. Peichl P, Holzer LA, Maier R, et al. Parathyroid hormone 1-84 accelerates fracture-healing in pubic bones of elderly osteoporotic women. J Bone Joint Surg Am. 2011;93(17):1583-1587.

23. Aspenberg P, Genant HK, Johansson T, et al. Teriparatide for acceleration of fracture repair in humans: a prospective, randomized, double-blind study of 102 postmenopausal women with distal radial fractures. $J$ Bone Miner Res. 2010;25(2):404-414.

24. Gomberg SJ, Wustrack RL, Napoli N, et al. Teriparatide, vitamin $\mathrm{D}$, and calcium healed bilateral subtrochanteric stress fractures in a postmenopausal woman with a 13-year history of continuous alendronate therapy. J Clin Endocrinol Metab. 2011;96(6):1627-1632.

25. Egol KA, Park JH, Prensky C, et al. Surgical treatment improves clinical and functional outcomes for patients who sustain incomplete bisphosphonate-related femur fractures. $J$ Orthop Trauma. 2013;27(6):331-335. 